\title{
Positive Periodic Solutions for First-Order Neutral Functional Differential Equations with Periodic Delays
}

\author{
Zeqing Liu, ${ }^{1}$ Xin Li, ${ }^{1}$ Shin Min Kang, ${ }^{2}$ and Young Chel Kwun ${ }^{3}$ \\ ${ }^{1}$ Department of Mathematics, Liaoning Normal University, Dalian, Liaoning 116029, China \\ ${ }^{2}$ Department of Mathematics and RINS, Gyeongsang National University, Jinju 660-701, Republic of Korea \\ ${ }^{3}$ Department of Mathematics, Dong-A University, Pusan 614-714, Republic of Korea
}

Correspondence should be addressed to Young Chel Kwun, yckwun@dau.ac.kr

Received 15 February 2012; Accepted 20 March 2012

Academic Editor: István Györi

Copyright (C) 2012 Zeqing Liu et al. This is an open access article distributed under the Creative Commons Attribution License, which permits unrestricted use, distribution, and reproduction in any medium, provided the original work is properly cited.

In this paper, two classes of first-order neutral functional differential equations with periodic delays are considered. Some results on the existence of positive periodic solutions for the equations are obtained by using the Krasnoselskii fixed point theorem. Four examples are included to illustrate our results.

\section{Introduction and Preliminaries}

In recent years, there have been a few papers written on the existence of periodic solutions, nontrivial periodic solutions, maximal and minimal periodic solutions and positive periodic solutions for several classes of functional differential equations with periodic delays, which arise from a number of mathematical ecological models, economical and control models, physiological and population models, and other models, see, for example, [1-5] and the references therein.

In 2004, Wan et al. [5] studied the first-order functional differential equation with periodic delays

$$
x^{\prime}(t)=-a(t) x(t)+f(t, x(t-\tau(t))), \quad \forall t \in \mathbb{R},
$$

where $a \in C\left(\mathbb{R}, \mathbb{R}^{+} \backslash\{0\}\right), \tau \in C(\mathbb{R}, \mathbb{R})$ are $\omega$-periodic, and $f \in C\left(\mathbb{R} \times \mathbb{R}^{+}, \mathbb{R}^{+}\right)$is $\omega$-periodic with respect to the first variable. By using a fixed point theorem in cones, they proved the 
existence of a periodic solution and a positive periodic solution of (1.1), respectively, under certain conditions. In 2005, Kang and Zhang [2] used the partial ordering and topological degree theory to establish the existence of a nontrivial periodic solution of (1.1). In 2010, Kang et al. [1] gave the existence of maximal and minimal periodic solutions of (1.1) by utilizing the method of lower and upper solutions. By means of the continuation theorem of coincidence degree principle, Serra [4] discussed the existence of periodic solutions for the following neutral functional differential equation

$$
[x(t)+c x(t-\tau)]^{\prime}=f(t, x(t)), \quad \forall t \in \mathbb{R},
$$

where $|c| \leq 1$ and $\tau>0$ are constants. In 2008, Luo et al. [3] employed the Krasnoselskii fixed point theorem to prove the existence of positive periodic solutions for two kinds of neutral functional differential equations with periodic delays

$$
\begin{gathered}
{[x(t)-c x(t-\tau(t))]^{\prime}=-a(t) x(t)+f(t, x(t-\tau(t))), \quad \forall t \in \mathbb{R},} \\
{\left[x(t)-c \int_{-\infty}^{0} Q(r) x(t+r) d r\right]^{\prime}=-a(t) x(t)+b(t) \int_{-\infty}^{0} Q(r) f(t, x(t+r)) d r, \quad \forall t \in \mathbb{R},}
\end{gathered}
$$

where $\omega \in \mathbb{R}^{+} \backslash\{0\}$ and $|c|<1$ are constants, $\tau \in C(\mathbb{R}, \mathbb{R}), a, b \in C\left(\mathbb{R}, \mathbb{R}^{+} \backslash\{0\}\right), f \in C\left(\mathbb{R}^{2}, \mathbb{R}\right)$, $\tau, a$, and $b$ are $\omega$-periodic and $f$ is $\omega$-periodic with respect to the first variable, $Q \in C\left(\mathbb{R}_{-}, \mathbb{R}^{+}\right)$, and $\int_{-\infty}^{0} Q(r) d r=1$.

Motivated by the papers [1-5] and the references therein, we consider two new kinds of first-order neutral functional differential equations with periodic delays:

$$
\begin{gathered}
{[g(t)(x(t)+c(t) x(t-\tau(t)))]^{\prime}=-a(t) x(t)+f(t, x(t-\tau(t))), \quad \forall t \in \mathbb{R},} \\
{\left[g(t)\left(x(t)+c(t) \int_{-\infty}^{0} Q(r) x(t+h(r)) d r\right)\right]^{\prime}} \\
\quad=-a(t) x(t)+b(t) \int_{-\infty}^{0} Q(r) f(t, x(t+h(r))) d r, \quad \forall t \in \mathbb{R},
\end{gathered}
$$

where $\omega \in \mathbb{R}^{+} \backslash\{0\}$ is a constant, $\tau, a, b, c \in C(\mathbb{R}, \mathbb{R}), f \in C\left(\mathbb{R}^{2}, \mathbb{R}\right), h \in C\left(\mathbb{R}_{-}, \mathbb{R}\right)$, $g \in C^{1}\left(\mathbb{R}, \mathbb{R}^{+} \backslash\{0\}\right), \tau, a, b, c$, and $g$ are $\omega$-periodic functions and $f$ is $\omega$-periodic with respect to the first variable, $Q \in C\left(\mathbb{R}_{-}, \mathbb{R}^{+}\right)$, and $\int_{-\infty}^{0} Q(r) d r=1$. It is evident that (1.4) and (1.5) include, respectively, (1.1)-(1.3) as special cases. To the best of our knowledge, the existence of periodic solutions for (1.4) and (1.5) have not been investigated till now. The aim of this paper is, by applying the Krasnoselskii fixed point theorem and some new techniques, to establish a set of sufficient conditions which guarantee the existence of positive periodic solutions of (1.4) and (1.5). Four examples are given to show the efficiency and applications of our results. 
Throughout this paper, we assume that $\mathbb{R}=(-\infty,+\infty), \mathbb{R}^{+}=[0,+\infty), \mathbb{R}_{-}=(-\infty, 0], \mathbb{N}$ denotes the set of all positive integers, $P=\min _{t \in[0, \omega]} g(t)$,

$$
\begin{gathered}
G(t, s)=\frac{\exp \left(\int_{t}^{s}\left(\left(g^{\prime}(r)+a(r)\right) / g(r)\right) d r\right)}{g(s)\left[\exp \left(\int_{0}^{\omega}\left(\left(g^{\prime}(r)+a(r)\right) / g(r)\right) d r\right)-1\right]}, \quad \forall(t, s) \in \mathbb{R}^{2}, \\
X=\{x \in C(\mathbb{R}, \mathbb{R}): x(t)=x(t+\omega), \forall t \in \mathbb{R}\} .
\end{gathered}
$$

It is well known that $X$ is a Banach space with the norm

$$
\|x\|=\sup _{t \in[0, \omega]}|x(t)|, \quad \text { for each } x \in X
$$

Let

$$
A(N, M)=\{x \in X: N \leq x(t) \leq M, \forall t \in[0, \omega]\}, \quad \text { for any } M>N \geq 0
$$

It is easy to see that $A(N, M)$ is a bounded closed and convex subset of the Banach space $X$.

Lemma 1.1 (the Krasnoselskii fixed point theorem). Let $Y$ be a nonempty bounded closed convex subset of a Banach space $Z$ and $f, g$ mappings from $Y$ into $Z$ such that $f x+g y \in Y$ for every pair $x, y \in Y$. If $f$ is a contraction mapping and $g$ is completely continuous, then the equation $f x+g x=x$ has at least one solution in $Y$.

\section{Main Results}

Now we use the Krasnoselskii fixed point theorem to show the existence of positive solutions for (1.4) and (1.5).

Theorem 2.1. Assume that there exist constants $N, M, G_{1}, G_{2}, c_{1}$, and $c_{2}$ satisfying

$$
\begin{gathered}
0<N<M, \quad c_{1} \geq 0, \quad c_{2} \geq 0, \quad c_{1}+c_{2}<1, \quad-c_{1} \leq c(t) \leq c_{2}, \quad \forall t \in[0, \omega], \\
0<G_{1} \leq g^{\prime}(t)+a(t) \leq G_{2}, \quad \forall t \in[0, \omega] \\
\left(N+c_{2} M\right) G_{2} \leq f(t, s)+a(t) c(t) s \leq\left(1-c_{1}\right) M G_{1}, \quad \forall(t, s) \in[0, \omega] \times[N, M] .
\end{gathered}
$$

Then (1.5) has at least one positive w-periodic solution in $A(N, M)$.

Proof. It is obvious that (1.4) has a solution $x(t)$ if and only if the integral equation

$$
x(t)=\int_{t}^{t+\omega} G(t, s)[f(s, x(s-\tau(s)))+a(s) c(s) x(s-\tau(s))] d s-c(t) x(t-\tau(t)), \quad \forall t \in \mathbb{R},
$$


has a solution $x(t)$. Define two mappings $T$ and $S: A(N, M) \rightarrow X$ by

$$
\begin{gathered}
(T x)(t)=\int_{t}^{t+\omega} G(t, s)[f(s, x(s-\tau(s)))+a(s) c(s) x(s-\tau(s))] d s, \quad \forall t \in \mathbb{R}, \\
(S x)(t)=-c(t) x(t-\tau(t)), \quad \forall t \in \mathbb{R}
\end{gathered}
$$

for each $x \in A(N, M)$. It follows from (2.5) that for any $x \in A(N, M)$ and $t \in \mathbb{R}$

$$
\begin{gathered}
(T x)(t+\omega)=\int_{t+\omega}^{t+2 \omega} G(t+\omega, s)[f(s, x(s-\tau(s)))+a(s) c(s) x(s-\tau(s))] d s \\
=\int_{t}^{t+\omega} G(t+\omega, u+\omega)[f(u+\omega, x(u+\omega-\tau(u+\omega))) \\
\quad+a(u+\omega) c(u+\omega) x(u+\omega-\tau(u+\omega))] d u \\
=\int_{t}^{t+\omega} G(t, u)[f(u, x(u-\tau(u)))+a(u) c(u) x(u-\tau(u))] d u=(T x)(t), \\
(S x)(t+\omega)=-c(t+\omega) x(t+\omega-\tau(t+\omega))=-c(t) x(t-\tau(t))=(S x)(t),
\end{gathered}
$$

which mean that

$$
T(A(N, M)) \subseteq X, \quad S(A(N, M)) \subseteq X
$$

Using (2.1)-(2.3) and (2.5), we infer that for all $x, y \in A(N, M)$ and $t \in \mathbb{R}$

$$
\begin{aligned}
(T x)(t)+(S y)(t)= & \int_{t}^{t+\omega} G(t, s)[f(s, x(s-\tau(s)))+a(s) c(s) x(s-\tau(s))] d s-c(t) y(t-\tau(t)) \\
\leq & \left(1-c_{1}\right) M G_{1} \int_{t}^{t+\omega} G(t, s) d s+c_{1} M \\
\leq & \left(1-c_{1}\right) M \int_{t}^{t+\omega} G(t, s)\left[g^{\prime}(s)+a(s)\right] d s+c_{1} M \\
= & \frac{\left(1-c_{1}\right) M}{\left[\exp \left(\int_{0}^{\omega}\left(\left(g^{\prime}(r)+a(r)\right) / g(r)\right) d r\right)-1\right]} \\
& \times \int_{t}^{t+\omega} \exp \left(\int_{t}^{s}\left(\frac{g^{\prime}(r)+a(r)}{g(r)}\right) d r\right)\left(\frac{g^{\prime}(s)+a(s)}{g(s)}\right) d s+c_{1} M \\
= & \frac{\left(1-c_{1}\right) M}{\left[\exp \left(\int_{0}^{\omega}\left(\left(g^{\prime}(r)+a(r)\right) / g(r)\right) d r\right)-1\right]} \\
& \times\left[\exp \left(\int_{t}^{t+\omega}\left(\frac{g^{\prime}(r)+a(r)}{g(r)}\right) d r\right)-1\right]+c_{1} M \\
= & \left(1-c_{1}\right) M+c_{1} M=M,
\end{aligned}
$$




$$
\begin{gathered}
(T x)(t)+(S y)(t) \geq\left(N+c_{2} M\right) G_{2} \int_{t}^{t+\omega} G(t, s) d s-c_{2} M \geq N+c_{2} M-c_{2} M=N, \\
|(S x)(t)-(S y)(t)|=|c(t)||x(t-\tau(t))-y(t-\tau(t))| \leq\left(c_{1}+c_{2}\right)\|x-y\|,
\end{gathered}
$$

which imply that

$$
T x+S y \in A(N, M), \quad\|S x-S y\| \leq\left(c_{1}+c_{2}\right)\|x-y\|, \quad \forall x, y \in A(N, M)
$$

Now we show that $T$ is a completely continuous mapping in $A(N, M)$. First, we claim that $T$ is continuous in $A(N, M)$. Let $\left\{y_{k}\right\}_{k \in N} \subset A(N, M)$ and $y \in A(N, M)$ with $\lim _{k \rightarrow \infty} y_{k}=$ $y$. Note that $f \in C\left(\mathbb{R}^{2}, \mathbb{R}\right)$. It follows from the uniform continuity of $f$ in $[0, \omega] \times[N, M]$ that for given $\varepsilon>0$, there exists $\delta>0$ satisfying

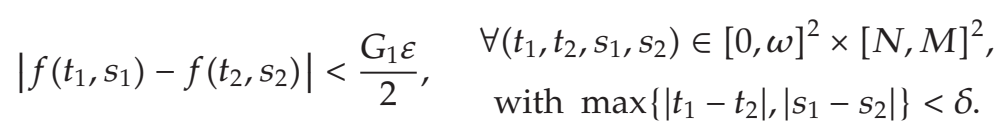

Since $\lim _{k \rightarrow \infty} y_{k}=y$, it follows that there exists $N_{1} \in \mathbb{N}$ satisfying

$$
\left\|y_{k}-y\right\|<\frac{G_{1} \min \{\varepsilon, \delta\}}{2\left(1+G_{2}\right)(1+\|a\|)}, \quad \forall k \geq N_{1}
$$

In view of (2.1), (2.2), (2.5), (2.12), and (2.13), we get that

$$
\begin{aligned}
\left\|T y_{k}-T y\right\| & =\sup _{t \in[0, \omega]} \mid \int_{t}^{t+\omega} G(t, s)\left[f\left(s, y_{k}(s-\tau(s))\right)+a(s) c(s) y_{k}(s-\tau(s))\right] d s \\
& -\int_{t}^{t+\omega} G(t, s)[f(s, y(s-\tau(s)))+a(s) c(s) y(s-\tau(s))] d s \mid \\
\leq & \sup _{t \in[0, \omega]} \int_{t}^{t+\omega} G(t, s)\left[\left|f\left(s, y_{k}(s-\tau(s))\right)-f(s, y(s-\tau(s)))\right|\right. \\
& \left.+\left|a(s) c(s) \| y_{k}(s-\tau(s))-y(s-\tau(s))\right|\right] d s \\
< & <G_{1}\left[\frac{\varepsilon}{2}+\frac{\|a\|\left(c_{1}+c_{2}\right) \min \{\varepsilon, \delta\}}{2\left(1+G_{2}\right)(1+\|a\|)}\right] \sup _{t \in[0, \omega]} \int_{t}^{t+\omega} G(t, s) d s<\varepsilon, \quad \forall k \geq N_{1},
\end{aligned}
$$

which yields that $\lim _{k \rightarrow \infty} T y_{k}=T y$, that is, $T$ is continuous in $A(N, M)$. 
Second, we claim that $T(A(N, M))$ is relatively compact. It is sufficient to show that $T(A(N, M))$ is uniformly bounded and equicontinuous in $[0, \omega]$. Notice that $(2.1)-(2.3)$ and (2.5) ensure that

$$
\begin{aligned}
\|T x\|= & \sup _{t \in[0, \omega]}\left|\int_{t}^{t+\omega} G(t, s)[f(s, x(s-\tau(s)))+a(s) c(s) x(s-\tau(s))] d s\right| \\
\leq & \left(1-c_{1}\right) M G_{1} \sup _{t \in[0, \omega]} \int_{t}^{t+\omega} G(t, s) d s \leq\left(1-c_{1}\right) M, \quad \forall x \in A(N, M), \\
\left|(T x)^{\prime}(t)\right|= & \mid-\frac{g^{\prime}(t)+a(t)}{g(t)}(T x)(t)+G(t, t+\omega) \\
& \times[f(t+\omega, x(t+\omega-\tau(t+\omega)))+a(t+\omega) c(t+\omega) x(t+\omega-\tau(t+\omega))] \\
& -G(t, t)[f(t, x(t-\tau(t)))+a(t) c(t) x(t-\tau(t))] \mid \\
\leq & \frac{g^{\prime}(t)+a(t)}{g(t)}|(T x)(t)|+|G(t, t+\omega)-G(t, t) \| f(t, x(t-\tau(t)))+a(t) c(t) x(t-\tau(t))| \\
\leq & \frac{G_{2}}{P}\left(1-c_{1}\right) M+\frac{\exp \left(\int_{0}^{\omega}\left(\left(g^{\prime}(r)+a(r)\right) / g(r)\right) d r\right)-1}{g(t)\left[\exp \left(\int_{0}^{\omega}\left(\left(g^{\prime}(r)+a(r)\right) / g(r)\right) d r\right)-1\right]}\left(1-c_{1}\right) M G_{1} \\
\leq & \frac{\left(1-c_{1}\right) M\left(G_{1}+G_{2}\right)}{P}, \quad \forall(x, t) \in A(N, M) \times[0, \omega]
\end{aligned}
$$

which give that $T(A(N, M))$ is uniformly bounded and equicontinuou sin $[0, \omega]$, which together with (2.7), (2.11), and Lemma 1.1 yields that there is $x_{0} \in A(N, M)$ with $T x_{0}+S x_{0}=$ $x_{0}$. It follows from (2.4) and (2.5) that $x_{0}$ is a positive $\omega$-periodic solution of (1.4). This completes the proof.

Theorem 2.2. Assume that there exist constants $N, M, G_{1}, G_{2}, c_{1}, c_{2}$, and $t_{0} \in[0, \omega]$ satisfying (2.2), (2.3):

$$
0 \leq N<M, \quad c_{1} \geq 0, \quad c_{2} \geq 0, \quad c_{1}+c_{2}<1, \quad-c_{1} \leq c(t) \leq c_{2}, \quad \forall t \in[0, \omega],
$$

and either

$$
f\left(t_{0}, s\right)+a\left(t_{0}\right) c\left(t_{0}\right) s>\left(N+c_{2} M\right) G_{2}, \quad \forall s \in[N, M]
$$

or

$$
g^{\prime}\left(t_{0}\right)+a\left(t_{0}\right)<G_{2}
$$

Then (1.4) has at least one positive w-periodic solution $x \in A(N, M)$ with $N<x(t) \leq M$ for each $t \in[0, \omega]$. 
Proof. As in the proof of Theorem 2.1, we conclude similarly that (1.4) has an w-periodic solution $x \in A(N, M)$. Now we assert that $x(t)>N$ for all $t \in[0, \omega]$. Otherwise, there exists $t^{*} \in[0, \omega]$ satisfying $x\left(t^{*}\right)=N$. In view of (2.4), (2.5), and (2.16), we have

$$
\begin{aligned}
N & =\int_{t^{*}}^{t^{*}+\omega} G\left(t^{*}, s\right)[f(s, x(s-\tau(s)))+a(s) c(s) x(s-\tau(s))] d s-c\left(t^{*}\right) x\left(t^{*}-\tau\left(t^{*}\right)\right) \\
& \geq \int_{t^{*}}^{t^{*}+\omega} G\left(t^{*}, s\right)[f(s, x(s-\tau(s)))+a(s) c(s) x(s-\tau(s))] d s-c_{2} M,
\end{aligned}
$$

which implies that

$$
\begin{aligned}
0 & \geq \int_{t^{*}}^{t^{*}+\omega} G\left(t^{*}, s\right)[f(s, x(s-\tau(s)))+a(s) c(s) x(s-\tau(s))] d s-\left(N+c_{2} M\right) \\
& =\int_{t^{*}}^{t^{*}+\omega} G\left(t^{*}, s\right)\left[f(s, x(s-\tau(s)))+a(s) c(s) x(s-\tau(s))-\left(N+c_{2} M\right)\left(g^{\prime}(s)+a(s)\right)\right] d s .
\end{aligned}
$$

Assume that (2.17) holds. By means of (2.2), (2.3), (2.17), and the continuity of $G, f, a$, $c, g, g^{\prime}, \tau$, and $x$, we get that

$$
\begin{gathered}
\int_{t^{*}}^{t^{*}+\omega} G\left(t^{*}, s\right)\left[f(s, x(s-\tau(s)))+a(s) c(s) x(s-\tau(s))-\left(N+c_{2} M\right)\left(g^{\prime}(s)+a(s)\right)\right] d s \\
\quad \geq \int_{t^{*}}^{t^{*}+\omega} G\left(t^{*}, s\right)\left[f(s, x(s-\tau(s)))+a(s) c(s) x(s-\tau(s))-\left(N+c_{2} M\right) G_{2}\right] d s>0
\end{gathered}
$$

which contradicts (2.20).

Assume that (2.18) holds. In light of (2.2), (2.3), (2.18), and the continuity of $G, f, a, c$, $g, g^{\prime}, \tau$, and $x$, we infer that

$$
\begin{aligned}
\int_{t^{*}}^{t^{*}+\omega} & G\left(t^{*}, s\right)\left[f(s, x(s-\tau(s)))+a(s) c(s) x(s-\tau(s))-\left(N+c_{2} M\right)\left(g^{\prime}(s)+a(s)\right)\right] d s \\
> & \int_{t^{*}}^{t^{*}+\omega} G\left(t^{*}, s\right)\left[f(s, x(s-\tau(s)))+a(s) c(s) x(s-\tau(s))-\left(N+c_{2} M\right) G_{2}\right] d s \geq 0
\end{aligned}
$$

which contradicts (2.20). This completes the proof.

Theorem 2.3. Assume that there exist constants $N, M, G_{1}, G_{2}, c_{1}$, and $c_{2}$ satisfying (2.1), (2.2), and

$$
\left(N+c_{2} M\right) G_{2} \leq b(t) f(t, s)+a(t) c(t) s \leq\left(1-c_{1}\right) M G_{1}, \quad \forall(t, s) \in[0, \omega] \times[N, M] .
$$

Then (1.5) has at least one positive w-periodic solution in $A(N, M)$. 
Proof. It is obvious that (1.5) has a solution $x(t)$ if and only if the integral equation

$$
\begin{aligned}
x(t)= & \int_{t}^{t+\omega} G(t, s)\left[b(s) \int_{-\infty}^{0} Q(r) f(s, x(s+h(r))) d r+a(s) c(s) \int_{-\infty}^{0} Q(r) x(s+h(r)) d r\right] d s \\
& -c(t) \int_{-\infty}^{0} Q(r) x(t+h(r)) d r, \quad \forall t \in \mathbb{R},
\end{aligned}
$$

has a solution $x(t)$. Define two mappings $T$ and $S: A(N, M) \rightarrow X$ by

$$
\begin{gathered}
(T x)(t)=\int_{t}^{t+\omega} G(t, s)\left[b(s) \int_{-\infty}^{0} Q(r) f(s, x(s+h(r))) d r+a(s) c(s) \int_{-\infty}^{0} Q(r) x(s+h(r)) d r\right] d s, \\
(S x)(t)=-c(t) \int_{-\infty}^{0} Q(r) x(t+h(r)) d r,
\end{gathered}
$$

for each $(x, t) \in A(N, M) \times \mathbb{R}$. The rest of the proof is similar to that of Theorem 2.1, and is omitted. This completes the proof.

Theorem 2.4. Assume that there exist constants $N, M, G_{1}, G_{2}, c_{1}, c_{2}$, and $t_{0} \in[0, \omega]$ satisfying (2.2), (2.16), (2.23), and either (2.18) or

$$
b\left(t_{0}\right) f\left(t_{0}, s\right)+a\left(t_{0}\right) c\left(t_{0}\right) s>\left(N+c_{2} M\right) G_{2}, \quad \forall s \in[N, M] .
$$

Then (1.5) has at least one positive w-periodic solution $x \in A(N, M)$ with $N<x(t) \leq M$ for each $t \in[0, \omega]$.

The proof of Theorem 2.4 is similar to that of Theorems 2.2 and 2.3 and is omitted.

Remark 2.5. Even if $g(t) \equiv 1, c(t) \equiv c$ and $h(r)=r$ for all $r \in \mathbb{R}_{-}$, the conditions of Theorems 2.2 and 2.4 in this paper are different from these conditions of Theorems 2.1-2.4 in [3], respectively.

\section{Examples}

Now we construct four examples which illustrate the results obtained in Section 2. Note that none of the known results can be applied to the examples.

Example 3.1. Consider the first-order neutral functional differential equation with periodic delays

$$
\begin{aligned}
& {\left[\left(1+\frac{\cos t}{100}\right)\left(x(t)+\frac{1+2 \sin t}{100} x(t-3 \sin t-2 \cos t)\right)\right]^{\prime}} \\
& \quad=-\left(1+\frac{\sin t}{50}\right) x(t)+20+\cos ^{2} t+\sin ^{2}\left(x^{5}(t-3 \sin t-2 \cos t) \cos t\right), \quad \forall t \in \mathbb{R} .
\end{aligned}
$$


Let $\omega=2 \pi, M=100, N=1, c_{1}=1 / 100, c_{2}=3 / 100, G_{1}=99 / 100, G_{2}=101 / 100$, and

$$
\begin{gathered}
g(t)=1+\frac{\cos t}{100}, \quad c(t)=\frac{1+2 \sin t}{100}, \quad a(t)=1+\frac{\sin t}{50}, \quad \tau(t)=3 \sin t+2 \cos t \\
f(t, s)=20+\cos ^{2} t+\sin ^{2}\left(s^{5} \cos t\right), \quad \forall(t, s) \in \mathbb{R}^{2} .
\end{gathered}
$$

It is easy to see that (2.1) and (2.2) hold. Notice that

$$
\begin{aligned}
\left(N+c_{2} M\right) G_{2} & =4.04<20+\left(1+\frac{1}{50}\right) \frac{1-2}{100} \cdot 100 \leq f(t, s)+a(t) c(t) s \\
& \leq 22+\left(1+\frac{1}{50}\right) \frac{1+2}{100} \cdot 100<98.01=\left(1-c_{1}\right) M G_{1}, \quad \forall(t, s) \in[0, \omega] \times[N, M],
\end{aligned}
$$

that is, (2.3) is satisfied. Thus Theorem 2.1 yields that (3.1) has a positive $\omega$-periodic solution in $A(N, M)$.

Example 3.2. Consider the first-order neutral functional differential equation with periodic delays

$$
\begin{aligned}
& {\left[\frac{3+2 \cos t+\sin t}{100}\left(x(t)+\frac{2+2 \sin t+\cos t}{20} x\left(t-\sin ^{3} t\right)\right)\right]^{\prime}} \\
& \quad=-\left(\frac{100+2 \sin t+3 \cos t}{100}\right) x(t)+60+\frac{x\left(t-\sin ^{3} t\right) \sin \sqrt{\left|t+x^{8}\left(t-\sin ^{3} t\right)\right|+1}}{50+10 \cos \left(t-x^{5}\left(t-\sin ^{3} t\right)\right)}, \quad \forall t \in \mathbb{R} .
\end{aligned}
$$

Let $\omega=2 \pi, M=100, N=0, c_{1}=1 / 20, c_{2}=1 / 4, G_{1}=24 / 25, G_{2}=26 / 25, t_{0}=\pi / 2$, and

$$
\begin{gathered}
g(t)=\frac{3+2 \cos t+\sin t}{100}, \quad c(t)=\frac{2+2 \sin t+\cos t}{20}, \quad \tau(t)=\sin ^{3} t, \\
a(t)=\frac{100+2 \sin t+3 \cos t}{100}, \quad f(t, s)=60+\frac{s \sin \sqrt{\left|t+s^{8}\right|+1}}{50+10 \cos \left(t-s^{5}\right)}, \quad \forall(t, s) \in \mathbb{R}^{2} .
\end{gathered}
$$

It is clear that (2.2), (2.16), and (2.18) hold. It follows that

$$
\begin{aligned}
\left(N+c_{2} M\right) G_{2} & =26<60+\frac{100(-1)}{50-10}+\frac{105}{100} \cdot \frac{-1}{20} \cdot 100 \leq f(t, s)+a(t) c(t) s \\
& \leq 60+\frac{100}{50-10}+26.25<91.2=\left(1-c_{1}\right) M G_{1}, \quad \forall(t, s) \in[0, \omega] \times[N, M],
\end{aligned}
$$


that is, (2.3) holds. Obviously (2.17) follows from the above inequalities. Hence, Theorem 2.2 ensures that (3.4) has a positive $\omega$-periodic solution $x \in A(N, M)$ with $N<x(t) \leq M$ for all $t \in[0, \omega]$

Example 3.3. Consider the first-order neutral functional differential equation with periodic delays

$$
\begin{aligned}
& {\left[\frac{5+\cos t+3 \sin t}{50+\sin t}\left(x(t)+\frac{2+3 \sin t}{6(50-\sin t)^{2}} \int_{-\infty}^{0} \exp (r) x(t-r \cos r) d r\right)\right]^{\prime}} \\
& =-\frac{151+50 \sin t}{(50+\sin t)^{2}} x(t)+\frac{1}{3+2 \sin t} \\
& \quad \times \int_{-\infty}^{0} \exp (r)\left[4.53+3 \sin t+\frac{3+2 \sin t}{10000+\cos t}\left[x(t-r \cos r) \cos ^{2} t+\cos \left(t+x^{100}(t-r \cos r)\right)\right]\right] d r,
\end{aligned}
$$

Let $\omega=2 \pi, M=1440.6, N=1, c_{1}=1 / 14406, c_{2}=5 / 14406, G_{1}=5 / 2601, G_{2}=295 / 2401$, and

$$
\begin{gathered}
g(t)=\frac{5+\cos t+3 \sin t}{50+\sin t}, \quad c(t)=\frac{2+3 \sin t}{6(50-\sin t)^{2}}, \quad a(t)=\frac{151+50 \sin t}{(50+\sin t)^{2}}, \quad b(t)=\frac{1}{3+2 \sin t}, \\
f(t, s)=4.53+3 \sin t+\frac{(3+2 \sin t)\left[s \cos ^{2} t+\cos \left(t+s^{100}\right)\right]}{10000+\cos t}, \quad \forall(t, s) \in \mathbb{R}^{2}, \\
Q(r)=\exp (r), \quad h(r)=-r \cos r, \quad \forall r \in \mathbb{R}_{-} .
\end{gathered}
$$

Clearly, (2.1) and (2.2) hold. Note that

$$
\begin{aligned}
\left(N+c_{2} M\right) G_{2} & =\frac{885}{4802}<\frac{4.53+3}{3+2}+\frac{-1}{10000-1}+\frac{302-553}{37470006} \cdot 1440.6 \leq b(t) f(t, s)+a(t) c(t) s \\
& \leq \frac{4.53-3}{3-2}+\frac{1441.6}{10000-1}+\frac{1005}{37470006} \cdot 1440.6<\frac{14404}{5282} \\
& =\left(1-c_{1}\right) M G_{1}, \quad \forall(t, s) \in[0, \omega] \times[N, M]
\end{aligned}
$$

that is, (2.23) is fulfilled. Thus Theorem 2.3 yields that (3.7) has a positive $\omega$-periodic solution in $A(N, M)$. 
Example 3.4. Consider the first-order neutral functional differential equation with periodic delays

$$
\begin{aligned}
{\left[\ln (100+2 \sin t)\left(x(t)+\frac{2+3 \cos t}{100-2 \sin t} \int_{-\infty}^{0} \exp (r) x(t+r) d r\right)\right]^{\prime} } \\
=-\left(\frac{3}{100+2 \sin t}\right) x(t) \\
\quad+\left(1+\frac{\cos t+\sin t}{100}\right) \int_{-\infty}^{0} \exp (r)\left[4+\frac{2 x(t+r) \sin t+\cos ^{2} t}{5000+5 \sin ^{2}\left[t-\ln \left(1+x^{2}(t+r)\right)\right]}\right] d r, \quad \forall t \in \mathbb{R} .
\end{aligned}
$$

Let $\omega=2 \pi, M=1000, N=1, c_{1}=1 / 98, c_{2}=5 / 98, G_{1}=1 / 102, G_{2}=5 / 98, t_{0}=\pi / 2$, and

$$
\begin{gathered}
g(t)=\ln (100+2 \sin t), \quad c(t)=\frac{2+3 \cos t}{100-2 \sin t}, \quad a(t)=\frac{3}{100+2 \sin t}, \quad b(t)=1+\frac{\cos t+\sin t}{100}, \\
f(t, s)=4+\frac{2 s \sin t+\cos ^{2} t}{5000+5 \sin ^{2}\left[t-\ln \left(1+s^{2}\right)\right]}, \quad \forall(t, s) \in \mathbb{R}^{2}, \quad Q(r)=\exp (r), \quad h(r)=r, \quad \forall r \in \mathbb{R}_{-} .
\end{gathered}
$$

Obviously, (2.2), (2.16), and (2.18) hold. A simple calculation yields that

$$
\begin{aligned}
\left(N+c_{2} M\right) G_{2} & =\frac{12745}{4802}<\frac{336103}{104125}=\left(1+\frac{-1-1}{100}\right)\left(4+\frac{-2000+0}{5000}\right)+\frac{6-9}{10000-4} \cdot 1000 \\
& \leq b(t) f(t, s)+a(t) c(t) s \leq\left(1+\frac{1+1}{100}\right)\left(4+\frac{2000+1}{5000}\right)+\frac{6+9}{10000-4} \cdot 1000 \\
& =\frac{623563}{104125}<\frac{24250}{2499}=\left(1-c_{1}\right) M G_{1}, \quad \forall(t, s) \in[0, \omega] \times[N, M]
\end{aligned}
$$

that is, (2.23) holds. Clearly (2.26) follows from the above inequalities. Thus Theorem 2.4 ensures that (3.10) has a positive $\omega$-periodic solution $x \in A(N, M)$ with $N<x(t) \leq M$ for all $t \in[0, \omega]$.

\section{Acknowledgment}

This study was supported by research funds from the Dong-A University.

\section{References}

[1] S. Kang, B. Shi, and G. Q. Wang, "Existence of maximal and minimal periodic solutions for first-order functional differential equations," Applied Mathematics Letters, vol. 23, no. 1, pp. 22-25, 2010.

[2] S. Kang and G. Zhang, "Existence of nontrivial periodic solutions for first order functional differential equations," Applied Mathematics Letters, vol. 18, no. 1, pp. 101-107, 2005. 
[3] Y. Luo, W. Wang, and J. H. Shen, "Existence of positive periodic solutions for two kinds of neutral functional differential equations," Applied Mathematics Letters, vol. 21, no. 6, pp. 581-587, 2008.

[4] E. Serra, "Periodic solutions for some nonlinear differential equations of neutral type," Nonlinear Analysis, vol. 17, no. 2, pp. 139-151, 1991.

[5] A. Wan, D. Q. Jiang, and X. J. Xu, "A new existence theory for positive periodic solutions to functional differential equations," Computers \& Mathematics with Applications, vol. 47, no. 8-9, pp. 1257-1262, 2004. 


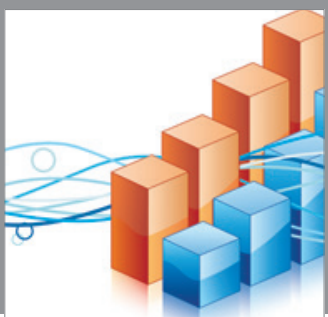

Advances in

Operations Research

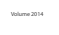

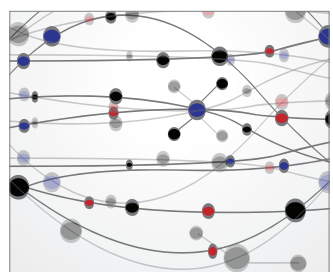

\section{The Scientific} World Journal
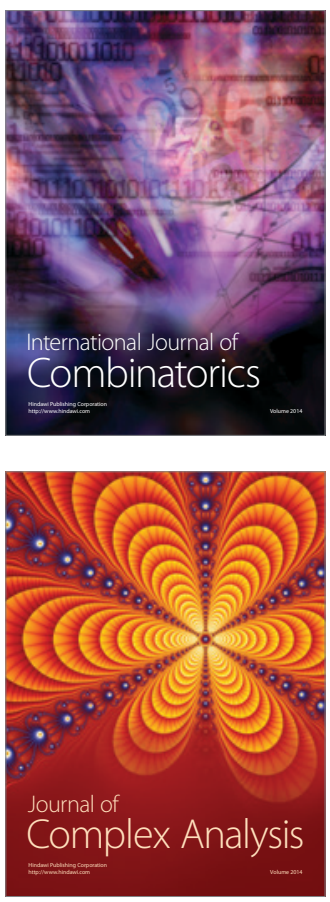

International Journal of

Mathematics and

Mathematical

Sciences
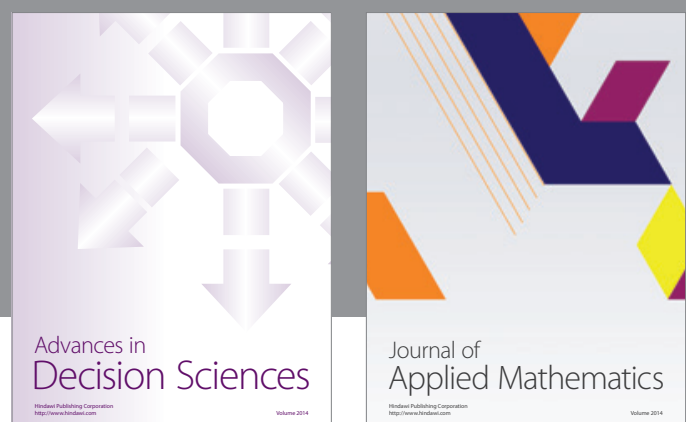

Journal of

Applied Mathematics
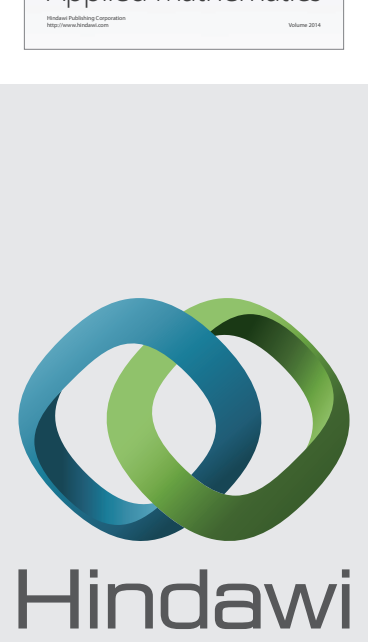

Submit your manuscripts at http://www.hindawi.com
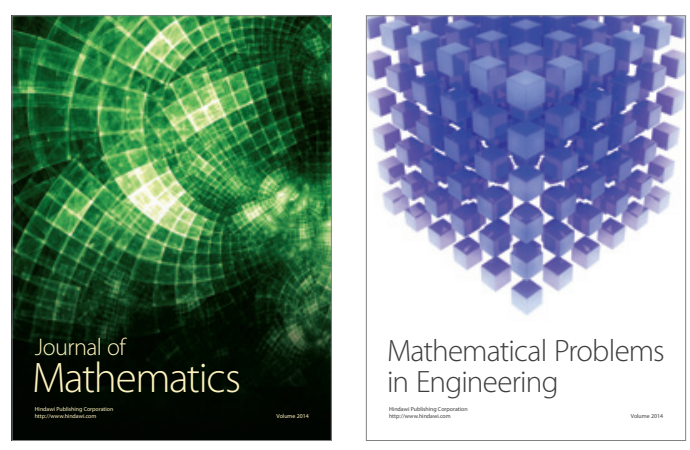

Mathematical Problems in Engineering
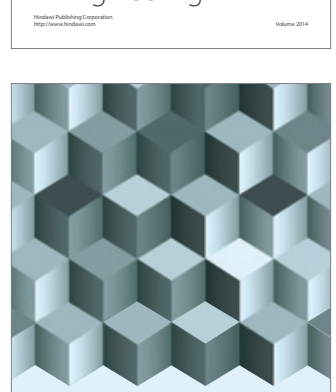

Journal of

Function Spaces
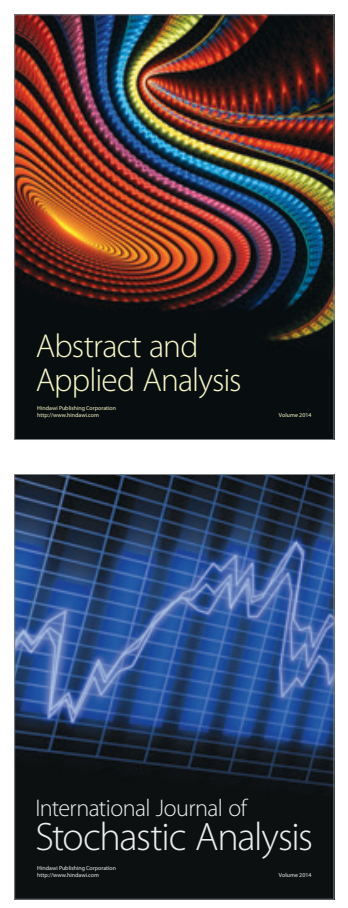

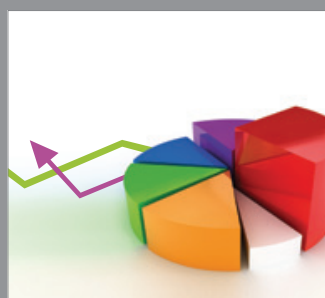

ournal of

Probability and Statistics

Promensencen
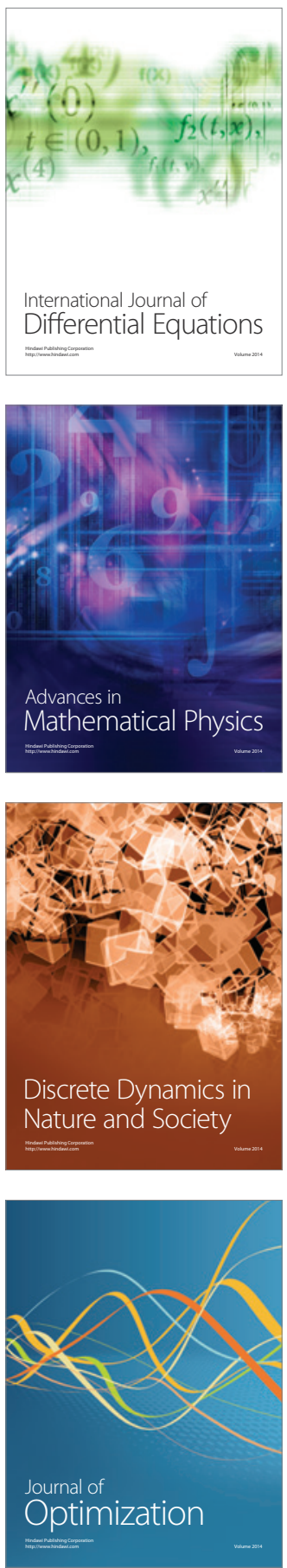